\title{
An Evolutionary Approach for Robust Layout Synthesis of MEMS
}

\author{
Fan, Zhun; Wang, Jiachuan; Goodman, Erik
}

Published in:

Advanced Intelligent Mechatronics. Proceedings, 2005 IEEE/ASME International Conference on

Link to article, DOI:

10.1109/AIM.2005.1511171

Publication date:

2005

Document Version

Publisher's PDF, also known as Version of record

Link back to DTU Orbit

Citation (APA):

Fan, Z., Wang, J., \& Goodman, E. (2005). An Evolutionary Approach for Robust Layout Synthesis of MEMS. In Advanced Intelligent Mechatronics. Proceedings, 2005 IEEE/ASME International Conference on IEEE. https://doi.org/10.1109/AIM.2005.1511171

\section{General rights}

Copyright and moral rights for the publications made accessible in the public portal are retained by the authors and/or other copyright owners and it is a condition of accessing publications that users recognise and abide by the legal requirements associated with these rights.

- Users may download and print one copy of any publication from the public portal for the purpose of private study or research.

- You may not further distribute the material or use it for any profit-making activity or commercial gain

- You may freely distribute the URL identifying the publication in the public portal 


\title{
An Evolutionary Approach For Robust Layout Synthesis of MEMS
}

\author{
Z. Fan, J. Wang, and E. Goodman, Member, IEEE
}

\begin{abstract}
The paper introduces a robust design method for layout synthesis of MEM resonators subject to inherent geometric uncertainties such as the fabrication error on the sidewall of the structure. The robust design problem is formulated as a multi-objective constrained optimisation problem after certain assumptions and treated with multiobjective genetic algorithm (MOGA), a special type of evolutionary computing approaches. Case study based on layout synthesis of a comb-driven MEM resonator shows that the approach proposed in this paper can lead to design results that meet the target performance and are less sensitive to geometric uncertainties than typical designs.
\end{abstract}

\section{INTRODUCTION}

$\mathrm{M}$ icro-electro-mechanical systems (MEMS) are tiny mechanical devices that are built onto semiconductor chips and are measured in micrometers. They usually integrate across different physical domains a number of functions, including fluidics, optics, mechanics and electronics, and are used to make devices such as pressure, temperature, chemical and vibration sensors, gyroscopes, engines, RF systems, and accelerometers for airbags.

Many designs of MEMS are made through engineering experience and back of the envelop calculations, and are highly dependent on designers' knowledge and experience. One reason for this is the complexity involved in the modeling, design and fabrication of MEMS - there are many constraints in designing and fabricating MEM devices due to the limitations of current fabrication techniques. As a result, many design issues are still not modeled and cannot be detected by the simulation software.

However, as process technologies become more stable, research emphasis has been shifted from developing specific process technologies towards the design of systems with a large number of reusable components, such as resonators, accelerometers, gyroscopes, and micro-mirrors. It is obvious that performance of individual components will influence the quality of the whole system [1]. For example, frequency stability of a MEM resonator can directly affect the quality of the MEM RF system in which it serves as a component of a filter or an oscillator.

Manuscript received February 28, 2005.

Z. Fan is with the Technical University of Denmark, Lyngby, DK 2800 Denmark (phone: 45452562 71; fax: 45459315 77; e-mail: zf@ mek.dtu.dk)

J. Wang is with the United Technologies Research Center, East Hartford, Connecticut, USA (e-mail: WangJ2@utrc.utc.com).

E. Goodman is with the Electrical and Computer Engineering Department, Michigan State University, East Lansing, MI 48823 USA (email: goodman@egr.msu.edu).
It greatly benefits the MEMS designers if the routine design of frequently used components can be optimized automatically by computer programs, while the designers can take more time in contemplating the more creative conceptual designs. The research of layout synthesis of microresonators has been carried out by many researchers. Some notable research include both deterministic numeric approaches [2]-[4] and meta-heuristic approaches such as evolutionary computation [5], [6] and simulated annealing [7] .

However, little has been done to account for the uncertainties and most of previous work has not considered another major dimension of MEMS design - robustness [12]. Actually, with current micromachining techniques, the fabrication process variation in MEMS is inevitable and will continue when devices are miniaturized to the point of process limitations [1]. For example, it is reported in [8] that the width of a typical suspension beam has a fabrication tolerance of about $10 \%$.

In this paper, we present a robust design approach for MEMS subject to process-induced geometrical uncertainties. In this approach, we first formulate the robust design problem as a multi-objective constrained optimization problem with two design objectives to be minimized, and then solve it using a multi-objective genetic algorithm (MOGA). The case study of robust layout synthesis of a comb-driven micro-resonator shows that the robust designs nominally meet the target performance and are less sensitive to geometric uncertainties.

\section{LAYOUT SYNTHESIS OF MEMS}

Layout synthesis automatically generates valid or optimized geometric sizing parameters for cell components, which in most cases are commonly used micromechanical devices with fixed topologies, according to engineering design objectives. The design objectives come from either high-level specifications such as behavioral model parameters that need to be satisfied, or from layout-level objectives such as minimum areas occupied. A normal approach is to model the design problem as a formal constrained optimization problem, and then solve it with powerful optimization techniques, resulting in a tool that automates the design synthesis of MEMS structures. Two categories of optimization techniques are used: one category includes stochastic algorithms such as genetic algorithms, and the other category includes deterministic algorithms such as nonlinear programming. For both categories, the process 
of solving the optimization problem involves determining the design variables, the design constraints, and the design objective. On top of that, design robustness receives more and more attentions recently, due to the fact that we can not avoid fabrication process variation in MEMS with current micromachining techniques.

\section{A. Design Variables and Constraints}

In this research, the cell component is a resonator device in MEMS domain. We decided to use 14 design variables for an example cell component, a folded-flexure comb-drive microresonator fabricated in a polysilicon surface microstructural process (Fig. 1, Fig. 2) [9] in this research.

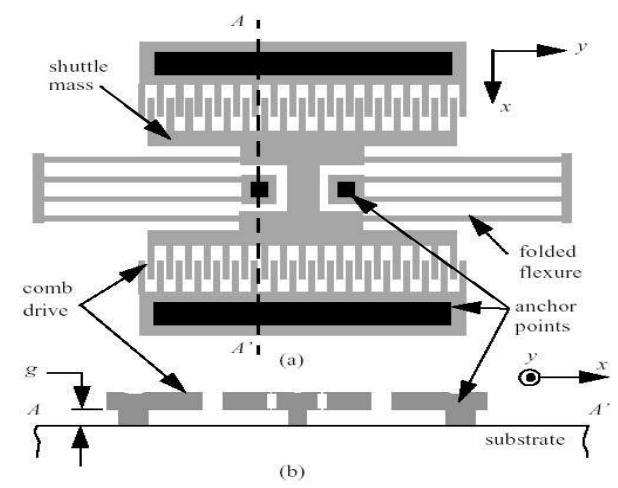

Fig. 1. A folded-flexure comb-drive microresonator fabricated in a polysilicon surface microstructural process a) Layout b) Crosssection A-A'
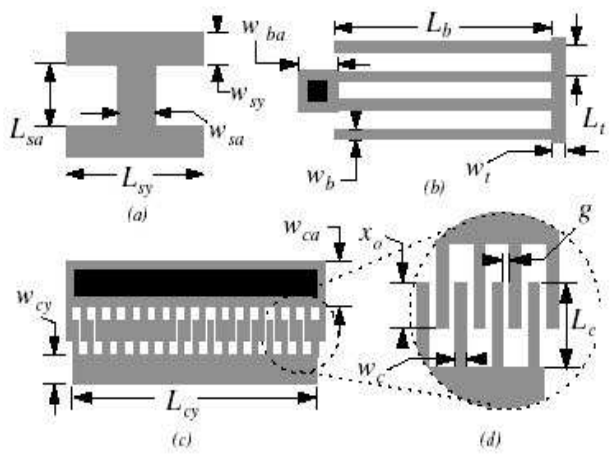

Fig. 2. Major design variables for the folded-flexure combdrive microresonator

Define design vector as follows:

$x=\left\{L_{b}, w_{b}, L_{t}, w_{t}, L_{s y}, w_{s y}\right.$,

$\left.w_{s a}, w_{c y}, L_{c y}, L_{c}, w_{c}, L_{s a}, x_{o}, V, N\right\}$

Design variables and their constraints are listed as follows:

$$
\begin{aligned}
& 2 \leq L_{b} \leq 400,2 \leq w_{b} \leq 20, \\
& 2 \leq L_{t} \leq 400,2 \leq w_{t} \leq 20, \\
& 2 \leq L_{s y} \leq 400,10 \leq w_{s y} \leq 400,
\end{aligned}
$$

$$
\begin{aligned}
& 10 \leq w_{s a} \leq 400,10 \leq w_{c y} \leq 400, \\
& 2 \leq L_{c y} \leq 700,8 \leq L_{c} \leq 400, \\
& 2 \leq w_{c} \leq 20,2 \leq L_{s a} \leq 400, \\
& 4 \leq x_{o} \leq 400,0 \leq V \leq 100,
\end{aligned}
$$

Note that the first 13 design variables have units of $\mu m$. The fourteenth design variable has units of volts. It is also important to note the role of feature size in VLSI and MEMS design. Feature size, which is often represented as $\lambda$, means the minimum size a particular design can achieve, based on specific fabrication procedures. In addition, the actual sizes of geometric shapes should be integer multiples of the feature size $\lambda$, such as $\lambda, 2 \lambda, 5 \lambda, 10 \lambda \ldots$ etc. In this research, we set $\lambda=0.09 \mu m$. This means that the first 13 design variables, which are geometric design variables, are actually not continuous variables. Instead, they are discretized by the feature size.

There are a number of design constraints for the microresonator cell component, including both geometric constraints and functional constraints. In this paper, without loss of generality, we consider the following constraints:

$$
\begin{aligned}
& g_{1}(x):-\left(L_{c y}+2 g+2 w_{c}\right) \leq 0 \\
& g_{2}(x): L_{c y}+2 g+2 w_{c}-700 \leq 0 \\
& g_{3}(x):-\left(L_{s y}+2 L_{b}+2 w_{t}\right) \leq 0 \\
& g_{4}(x): L_{s y}+2 L_{b}+2 w_{t}-700 \leq 0 \\
& \quad-\left(3 L_{t}+w_{s y}+4 L_{c}\right. \\
& \left.g_{5}(x): \quad-2 x_{0}+2 w_{c y}+2 w_{c a}\right) \leq 0 \\
& \quad 3 L_{t}+w_{s y}+4 L_{c}- \\
& g_{6}(x): \begin{array}{l}
2 x_{0}+2 w_{c y}+2 w_{c a}-700 \leq 0 \\
g_{7}(x):
\end{array} L_{c}-\left(x_{0}+x_{d i s p}\right)-200 \leq 0 \\
& g_{8}(x): 4-L_{c}+\left(x_{0}+x_{d i s p}\right) \leq 0
\end{aligned}
$$

Among them, the first six are linear constraints, but the last two are nonlinear constraints because the term $x_{d i s p}$ is highly nonlinear.

$$
x_{\text {disp }}=Q F_{e, x} / K_{x} \text {, }
$$

where

$$
F_{e, x}=1.12 \varepsilon_{0} N \frac{t}{g} V^{2},
$$

and

$$
K_{x}=\frac{2 E t W_{b}^{3}}{L_{b}{ }^{3}} \frac{L_{t}{ }^{2}+14 \alpha L_{t} L_{b}+36 \alpha^{2} L_{b}{ }^{2}}{4 L_{t}^{2}+41 \alpha L_{t} L_{b}+36 \alpha^{2} L_{b}{ }^{2}}
$$


where $\alpha=\left(W_{t} / W_{b}\right)^{3}$

and $m_{x}=m_{s}+\frac{1}{4} m_{t}+\frac{12}{35} m_{b}$

where $m_{s}=\rho A_{s}, m_{t}=\rho A_{t}, m_{b}=\rho A_{b}$

$A_{s}=w_{s a} L_{s a}+2 w_{s y} L_{s y}$

$A_{t}=2 w_{c a} L_{c y}$

$A_{b}=8 L_{b} w_{b}+2 w_{t}\left(2 L_{t}+w_{a}+2 w_{b}\right)$

The natural frequency $\omega_{n}$ is defined as

$\omega_{n}=\sqrt{\frac{K_{x}}{m_{x}}}$

\section{B. Design Objectives}

The design objective is to match the natural frequency $\omega_{n}(x, \delta)$ of the resonator with a predefined natural frequency, where $x \in \mathfrak{R}^{n}$ is the design vector, $\delta \in \mathfrak{R}^{n}$ is the uncertainty. If we represent the predefined frequency as $\omega_{t \text { arg } e t}$, then the design objective can be formulated as:

$$
\min _{x} \mathrm{E}\left(\omega_{n}(x, \delta)-\omega_{t \arg e t}\right)^{2}
$$

subject to $g_{i}(x) \leq 0$

where $\omega_{t \text { arg } e t}$ is the target natural frequency we would like the resonator to work at, and the expectation is taken over the random vector $\delta$. We assume that the uncertainty, $\delta$, can be characterized as a random vector with the following statistics

$$
\begin{aligned}
& \mathrm{E}(\delta)=0_{n x 1} \\
& \mathrm{E}\left(\delta \delta^{T}\right)=\Omega \in \mathfrak{R}^{n x n}
\end{aligned}
$$

where $\Omega$ is the covariance matrix and is positive semidefinite. If the uncertainties are uncorrelated then $\Omega$ is diagonal, otherwise the off-diagonal entries are non-zero when correlation exists.

\section{Modeling Uncertainty in MEMS}

In this research, we assume that the uncertainty in the fabrication process is introduced by etch-induced variations in line-width, and the structure is etched uniformly.

Fig. 3 illustrate the two uniform etch scenarios on a structure - overetch and underetch. Take the under-etch situation for example, after process variation is introduced, some design variables may increase (such as $\mathrm{L}_{1}$ and $\mathrm{L}_{2}$ ), other design variables (such as $\mathrm{L}_{3}$ ) may decrease, while some others may stay unchanged (such as $\mathrm{L}_{4}$ ).

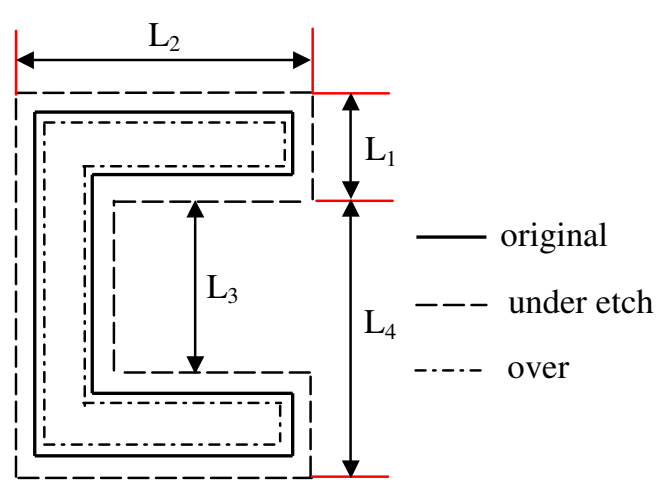

Fig. 3. Under- and over-etch of a MEM structure

We can model the geometric process variations using a simple additive uncertain model

$\tilde{x}=x+\delta$

where $\tilde{x}$ is the uncertain (actual) design vector and for the above simple example $\delta=\left\{\delta_{L_{1}}, \delta_{L_{2}}, \delta_{L_{3}}, \delta_{L_{4}}\right\}$.

Since the structure is etched uniformly, we can assume that the standard deviation of each term of $\delta$ is equal. If we define $\rho$ to be a normal random variable with standard deviation of $\sigma$, then we can write

$\delta=\rho \zeta$

where $\zeta=[1,1-1,0]^{T}$, note that in the condition of underetch, $\mathrm{L}_{1}$ and $\mathrm{L}_{2}$ increase, $\mathrm{L}_{3}$ decreases, and $\mathrm{L}_{4}$ is not changed.

According to (12), we can obtain

$\Omega=\mathrm{E}\left(\delta \delta^{T}\right)=\sigma^{2} \zeta \zeta^{T}$

For the case study of micro-resonator discussed in this paper,

$$
\begin{aligned}
& \delta=\left\{\begin{array}{l}
\delta_{L_{b}}, \delta_{w_{b}}, \delta_{L_{t}}, \delta_{w_{t}}, \delta_{L_{s y}}, \delta_{w_{s y}}, \delta_{w_{s a}}, \\
\delta_{w_{c y}}, \delta_{L_{c y}}, \delta_{L_{c}}, \delta_{w_{c}}, \delta_{L_{s a}}, \delta_{x_{o}}, \delta_{V}
\end{array}\right\} \\
& \text { and } \\
& \zeta=[0,1,-1,1,1,1,1,1,1,0,1,-1,1,0]^{T}
\end{aligned}
$$

\section{Robust Design Problem Formulation}

The problem posed in (10) is a difficult robust optimization problem to solve in general. To simplify the problem, we choose to approximate $\omega_{n}(x, \delta)$ with a first order Taylor series expansion in $\delta$ as

$\omega_{n}(x, \delta) \cong \omega_{n}(x, 0)+\nabla_{x} \omega_{n}(x, 0) \delta$

where $\nabla_{x} \omega_{n}(x, 0)$ is the gradient of $\omega_{n}(x, 0)$ with respect to $x$. Using this approximation, we can expand the expression of $\left(\omega_{n}(x, \delta)-\omega_{t \text { arg } e t}\right)^{2}$ into 


$$
\begin{aligned}
& \left(\omega_{n}(x, \delta)-\omega_{t \arg e t}\right)^{2} \cong\left(\omega_{n}(x, 0)-\omega_{t \arg e t}\right)^{2}+ \\
& 2\left(\omega_{n}(x, 0)-\omega_{t \arg e t}\right) \nabla_{x} \omega_{n}(x, 0) \delta+ \\
& \nabla_{x} \omega_{n}(x, 0) \delta \delta^{T} \nabla_{x}{ }^{T} \omega_{n}(x, 0)
\end{aligned}
$$

Taking the expectation of the above equation, we can get

$$
\begin{aligned}
& \mathrm{E}\left(\omega_{n}(x, \delta)-\omega_{t \arg e t}\right)^{2} \cong\left(\omega_{n}(x, 0)-\omega_{t \arg e t}\right)^{2}+ \\
& 2\left(\omega_{n}(x, 0)-\omega_{t \arg e t}\right) \nabla_{x} \omega_{n}(x, 0) \mathrm{E}(\delta)+ \\
& \nabla_{x} \omega_{n}(x, 0) \mathrm{E}\left(\delta \delta^{T}\right) \nabla_{x}{ }^{T} \omega_{n}(x, 0)
\end{aligned}
$$

By reducing equation (6), based on our assumptions about the mean and covariance of $\delta$ according to (11) and (12), we obtain

$$
\begin{aligned}
& \mathrm{E}\left(\omega_{n}(x, \delta)-\omega_{t \arg e t}\right)^{2} \cong\left(\omega_{n}(x, 0)-\omega_{t \arg e t}\right)^{2}+ \\
& \nabla_{x} \omega_{n}(x, 0) \Omega \nabla_{x}{ }^{T} \omega_{n}(x, 0)
\end{aligned}
$$

substituting the approximation in (19) back into the original design problem posed in (10) yields

$$
\min _{x}\left(\left(\omega_{n}(x, 0)-\omega_{t \text { arg } e t}\right)^{2}+\nabla_{x} \omega_{n}(x, 0) \Omega \nabla_{x}{ }^{T} \omega_{n}(x, 0)\right)
$$

subject to $g_{i}(x) \leq 0$

To non-dimensionalize the cost function, we decide to divide through by $\omega_{t \arg e t}{ }^{2}$. We then refer to the following expression as our robust design problem

$$
\min _{x}\left(\begin{array}{l}
\left(\frac{\omega_{n}(x, 0)-\omega_{t \arg e t}}{\omega_{t \arg e t}}\right)^{2}+ \\
\frac{1}{\omega_{t \text { arg } e t}^{2}}\left(\nabla_{x} \omega_{n}(x, 0) \Omega \nabla_{x}{ }^{T} \omega_{n}(x, 0)\right)
\end{array}\right)
$$

subject to $g_{i}(x) \leq 0$

It is now easy to see that the expression we want to minimize has two distinct terms. For notational convenience, we will label the two terms as

$$
\begin{aligned}
& N(x) \equiv\left(\frac{\omega_{n}(x, 0)-\omega_{t \arg e t}}{\omega_{t \arg e t}}\right)^{2} \\
& D(x, \Omega) \equiv \frac{1}{\omega_{t \text { arg } e t}^{2}} \nabla_{x} \omega_{n}(x, 0) \Omega \nabla_{x}^{T} \omega_{n}(x, 0)
\end{aligned}
$$

with the above definitions the robust design problem posed in (10) becomes

$$
\min _{x} N(x)+D(x, \Omega) \text { subject to } g_{i}(x) \leq 0
$$

(24) The first term, $N(x)$, penalizes deviation of the nominal solution, $\omega_{n}(x, 0)$, from the target, $\omega_{t \text { arg } e t}$, while the second term, $D(x, \Omega)$, penalizes the sensitivity of the design with respect to $\delta$. Since there are two objectives in the formation of the cost function to be minimized, a tradeoff is usually needed to be made by the designer to either focus on minimizing the squared error of the nominal design or on reducing the sensitivity.

\section{ROBUST DESIGN OPTIMIZATION USING AN EVOLUTIOANRY APPROACH}

\section{A. Multiobjective Genetic Algorithm}

Constrained multi-objective optimisation problems involving multiple constraints and more than two conflicting objectives are common within engineering, operations research and computer science communities. Most classical optimization methods usually convert multiple objectives into a single objective by using some subjective preference information. They are only capable of finding one optimal solution at a time and are usually required to be used repeatedly to find multiple trade-off solutions. The multiobjective genetic algorithm (MOGA) is a new, emerging and fast-growing field of research and application for solving such optimisation problems. MOGAs are capable of finding multiple trade-off optimal solutions in a single computer simulation. In particular, a simple yet efficient algorithm, called nondominated sorting genetic algorithm II (NSGA-II), has been extensively used recently, because of its simplicity, demonstrated superiority over other existing methods, and availability of a freely downloadable computer code [10]. With a fast nondominated sorting procedure, and a modified definition of dominance in order to solve constrained Multiobjective problems efficiently, NSGA-II is found to be able to find a spread of solutions that converge towards the true Pareto-optimal front in difficult test problems. It is also found that NSGA-II is capable of solving any number of objectives with an iteration-wise complexity of $O\left(M N^{2}\right)$, where $M$ is the number of objectives and $N$ is the number of solutions used in a NSGA-II population [11].

NSGA-II is useful in this research not only because the knowledge of multiple trade-off solutions helps a decisionmaker to make a better and more confident choice of a solution, but also because of its convenience to solve the optimisation problem with both discrete and continuous design variables. While it is very difficult for many numerical optimization approaches (for example, gradientbased approaches) to include considerations of feature size constraints [4], it is quite convenient for genetic algorithms to do so. We need to modify the objective function only slightly, mapping real values of design variables to integer multiples of the feature size $\lambda$ before using them in formulations of constraints and objectives. No modifications to the genetic algorithm are needed.

In this research, we have used the following parameter values:

Population size: $\quad 100$

Number of generations: $\quad 100$ 
Crossover probability:

0.9

Real-parameter mutation probability: 0.07

Distribution index for crossover: $\quad 10$

Distribution index for mutation: $\quad 50$

\section{B. Simulation results}

We apply NSGA-II to solve the two-objective optimization problem of minimizing the deviation of the nominal solution and sensitivity of the design. Design results for resonators with natural frequencies of $1000 \mathrm{~Hz}$ and $10,000 \mathrm{~Hz}$ are reported. Results with and without consideration of robust design are compared.

TABLE I

DESIGN RESULTS COMPARISONS

\begin{tabular}{|c|c|c|c|c|}
\hline & \multicolumn{2}{|c|}{$\omega_{n}=1 \mathrm{~K}$} & \multicolumn{2}{|c|}{$\omega_{n}=10 \mathrm{~K}$} \\
\hline & $\begin{array}{l}\text { Robust } \\
\text { Design }\end{array}$ & $\begin{array}{c}\text { Non- } \\
\text { Robust } \\
\text { Design } \\
\end{array}$ & $\begin{array}{l}\text { Robust } \\
\text { Design }\end{array}$ & $\begin{array}{c}\text { Non- } \\
\text { Robust } \\
\text { Design } \\
\end{array}$ \\
\hline$L_{b}(\mu m)$ & 209.61 & 204.66 & 69.75 & 53.37 \\
\hline$w_{b}(\mu m)$ & 12.69 & 13.59 & 19.89 & 9.54 \\
\hline$L_{t}(\mu m)$ & 168.21 & 136.53 & 99.36 & 44.82 \\
\hline$w_{t}(\mu m)$ & 4.05 & 4.59 & 19.8 & 2.43 \\
\hline$L_{s y}(\mu m)$ & 78.57 & 73.17 & 395.37 & 63.72 \\
\hline$w_{s y}(\mu m)$ & 325.89 & 77.22 & 272.61 & 143.55 \\
\hline$w_{s a}(\mu m)$ & 36.27 & 12.87 & 365.13 & 12.06 \\
\hline$w_{c y}(\mu m)$ & 253.17 & 150.21 & 372.96 & 96.48 \\
\hline$L_{c y}(\mu m)$ & 307.35 & 241.74 & 591.12 & 261.72 \\
\hline$L_{c}(\mu m)$ & 154.89 & 162.72 & 277.65 & 95.76 \\
\hline$w_{c}(\mu m)$ & 7.56 & 2.07 & 248.94 & 10.80 \\
\hline$L_{s a}(\mu m)$ & 104.22 & 243.36 & 172.26 & 76.95 \\
\hline$x_{o}(\mu m)$ & 150.57 & 142.2 & 38.97 & 54.72 \\
\hline$V($ volt $)$ & 10.00 & 11.00 & 98.00 & 10.00 \\
\hline $\begin{array}{l}\text { Deviation } \\
\text { of } \omega_{n}\end{array}$ & $3.53 \%$ & $6.33 \%$ & $1.66 \%$ & $3.65 \%$ \\
\hline
\end{tabular}

To demonstrate robustness of our solutions, we ran a Monto-Carlo simulation to model an uncertain MEMS fabrication process. To represent the variation in the process we generated 5,000 normal random vectors with a standard deviation of $2 \lambda$. We calculated the standard deviation of the frequencies subject to this variation. It can be seen from Table 1 that in both cases, the robust design has a much smaller (about 50\%) standard deviation than the non-robust design. Fig. 4. shows distributions of the resonant frequencies of the designs subject to the uncertainty. Fig. 5 shows a distribution of Pareto-optimal front NSGA-II obtained in searching for robust solutions of resonator design with frequency of $1000 \mathrm{~Hz}$. We can see that for some design solutions, the resulting resonator frequencies are closer to the nominal value, but their sensitivities to variations, $D(x, \Omega)$, are bigger. For some others, the deviations from the nominal solution, $N(x)$, are smaller, but the sensitivities are higher. It is difficult to declare which solutions are superior to the others. Or in other words, none of the solutions in the Pareto-optimal front dominates other solutions. It is up to the designer to make trade offs in real world design applications.

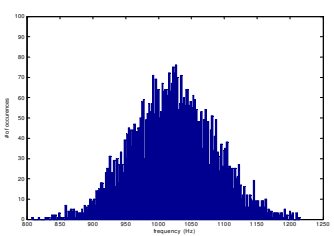

(a)

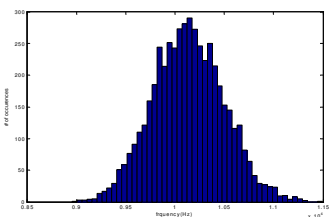

(c)

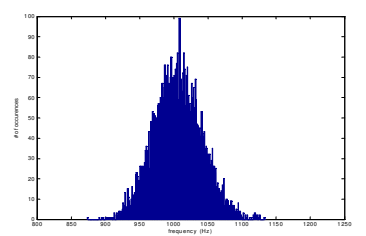

(b)

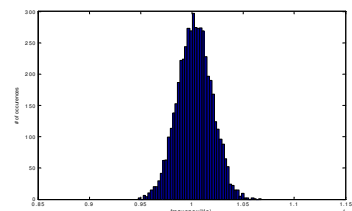

(d)
Fig. 4. Distributions of resonant frequencies subject to processinduced variations for non-robust and robust designs. The horizontal axis represents frequency, the vertical axis represents number of occurrences.

(a) non-robust resonator design with frequency $=1000 \mathrm{~Hz}$

(b) robust resonator design with frequency $=1000 \mathrm{~Hz}$

(c) non-robust resonator design with frequency $=10,000 \mathrm{~Hz}$

(d) robust resonator design with frequency $=10,000 \mathrm{~Hz}$ 


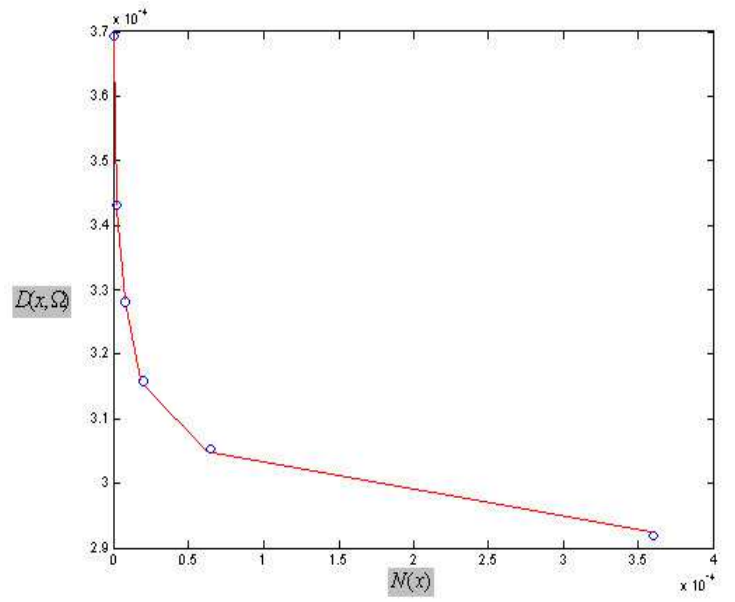

Fig. 5. NSGA-II solutions for the minimization of deviation of the nominal solution and sensitivity of the design

\section{CONCLUSIONS}

Layout synthesis is an important stage for structured design of MEMS [13], [14], after the stage of the systemlevel design [15]. The paper reports a method of robust layout synthesis of MEMS that transforms the robust design problem into a multi-objective constrained optimisation problem, and then solve it using NSGA-II, a special type of MOGA. Simulation results on a case study of surfacemachined comb-driven resonator show that the robust design solutions are less sensitive to process induced uncertainties. While it is important to study more efficient robust design method and more effective approaches to model uncertainties, it is an interesting research topic to investigate the relationship between the robustness subject to parametric variations (such as the layout synthesis) and the topologyrelated robustness in the system level design.

\section{REFERENCES}

[1] R. Liu, B. Paden, and K. Turner, "MEMS Resonators That are Robust to Process-Induced Feature Width Variations," Journal of Microelectromechanical Systems, vol. 11, No. 5, pp. 505511, October 2002.

[2] W. Yeh, S. Mukherjee, and N. C. MacDonald, "Optimal Shape Design of An Electrostatic Comb Drive in MEMS," $J$. Microelectromech. Syst. vol. 7, pp. 16-26, 1998.

[3] T. Mukherjee, S. Iyer and G. K. Feeder, "Optimization-based synthesis of micro-resonators," Sensors Actuators, A 70, pp. 118-127, 1998.

[4] G. Fedder, S. V. Iyer and T. Mukherjee, "Automated Optimal Synthesis Of Microresonators," in Proceedings of 9th International Conference on Solid State Sensors and Actuators (TRANSDUCERS '97), pp. 1109-1112, June 16-19, 1997, Chicago, IL, USA

[5] R. Kamalian, H. Takagi, and A. M. Agogino, "Optimized Design of MEMS by Evolutionary Multi-objective Optimization with Interactive Evolutionary Computation," in K. Deb et al. (Eds.): GECCO 2004, LNCS 3103, Springer-Verlag Berlin Heidelberg, pp. 1030-1041, 2004.

[6] L. Ma and E. K. Antonsson, "Automated Mask-Layout and Process Synthesis for MEMS," in Technical Proceedings of the
2000 International Conference on Modeling and Simulation of Microsystems, pp. 20-23, 2000.

[7] Ongkodjojo, F. E. H. Tay, "Global Optimization and Design for Microelectromechanical Systems devices based on Simulated Annealing," Journal of Micromechanics and Microengineering, vol. 12, pp. 878-897, 2002.

[8] Y. S. Hong, J. H. Lee, and S. H. Kim, "A laterally driven symmetric micro-resonator for gyroscopic applications," Journal of Micromechanics and Microengineering, vol. 10, pp 452-458, 2000.

[9] G. Fedder and T. Mukherjee, "Physical Design for SurfaceMicromachined MEMS," in Proceedings of the Fifth ACM/SIGDA Physical Design Workshop, pp. 53-60, April 1996.

[10] Available: http://www.iitk.ac.in/kangal/soft.htm

[11] K. Deb, A. Pratap, S. Agarwal, and T. Meyarivan, "A fast and elitist multiobjective genetic algorithm: NSGA-II," IEEE Transactions on Evolutionary Computation, vol. 6, No. 2, April 2002.

[12] G. Taguchi, Taguchi on Robust Technology Development: Bringing Quality Engineering Upstream, ASME Press, New York, 1993.

[13] T. Mukherjee and G. Fedder, "Structured Design Of Microelectromechanical Systems," in Proceedings of 34th Design Automation Conference (DAC '97), , Anaheim, CA, USA, pp. 680-685, June 9-13, 1997.

[14] G. Fedder, "A Vision of Structured CAD for MEMS," in Proceedings of the Fifth ACM/SIGDA Physical Design Workshop

[15] Z. Fan, K. Seo, R. Rosenberg, J. Hu, E. Goodman, "SystemLevel Synthesis of MEMS via Genetic Programming and Bond Graphs," in Proc. 2003 Genetic and Evolutionary Computing Conference, Chicago, Springer, Lecture Notes in Computer Science, pp. 2058-2071, July, 2003. 